\title{
Article \\ Theoretical Modeling and Analysis of Directional Spectrum Emissivity and Its Pattern for Random Rough Surfaces with a Matrix Method
}

\author{
Jianrui Hu ${ }^{1,2}$, Zhanqiang Liu ${ }^{1,2, * \mathbb{D}}$, Jinfu Zhao ${ }^{1,2}$, Bing Wang ${ }^{1,2}$ and Qinghua Song ${ }^{1,2} \mathbb{D}$ \\ 1 School of Mechanical Engineering, Shandong University, Jinan 250061, China; \\ sduhujianrui@mail.sdu.edu.cn (J.H.); zhaojinfu@mail.sdu.edu.cn (J.Z.); sduwangbing@sdu.edu.cn (B.W.); \\ ssinghua@sdu.edu.cn (Q.S.) \\ 2 Key National Demonstration Center for Experimental Mechanical Engineering Education/Key Laboratory of \\ High Efficiency and Clean Mechanical Manufacture of MQE, Jinan 250061, China \\ * Correspondence: melius@sdu.edu.cn
}

Citation: Hu, J.; Liu, Z.; Zhao, J.; Wang, B.; Song, Q. Theoretical

Modeling and Analysis of Directional Spectrum Emissivity and Its Pattern for Random Rough Surfaces with a Matrix Method. Symmetry 2021, 13, 1733. https://doi.org/10.3390/ sym13091733

Academic Editor: Angelo Freni

Received: 30 August 2021

Accepted: 15 September 2021

Published: 18 September 2021

Publisher's Note: MDPI stays neutral with regard to jurisdictional claims in published maps and institutional affiliations.

Copyright: (c) 2021 by the authors. Licensee MDPI, Basel, Switzerland. This article is an open access article distributed under the terms and conditions of the Creative Commons Attribution (CC BY) license (https:// creativecommons.org/licenses/by/ $4.0 /)$.

\begin{abstract}
The emissivity is an important surface property parameter in many fields, including infrared temperature measurement. In this research, a symmetry theoretical model of directional spectral emissivity prediction is proposed based on Gaussian random rough surface theory. A numerical solution based on a matrix method is determined based on its symmetrical characteristics. Influences of the index of refraction $n$ and the root mean square (RMS) roughness $\sigma_{r m s}$ on the directional spectrum emissivity $\varepsilon$ are analyzed and discussed. The results indicate that surfaces with higher $n$ and lower $\sigma_{r m s}$ tend to have a peak in high viewing angles. On the contrary, surfaces with lower $\mathrm{n}$ and higher $\sigma_{r m s}$ tend to have a peak in low viewing angles. Experimental verifications based on infrared (IR) temperature measurement of Inconel 718 sandblasted surfaces were carried out. This model would contribute to understand random rough surfaces and their emitting properties in fields including machining, process controlling, remote sensing, etc.
\end{abstract}

Keywords: Inconel 718; surface integrity; Gaussian random rough surface; matrix method; infrared emissivity

\section{Introduction}

As a surface property parameter, the precise determination of emissivity is important in many fields, including infrared temperature measurement. Experimental studies have shown that the main factors affecting the emissivity of a given rough surface are the chemical composition [1], temperature [2-4], wavelength [2,3] and surface morphology [4,5], as shown in Figure 1. Closely related to the speed of light in the material, the index of refraction $n$ depends mainly on the material, and is affected by temperature, wavelength and other factors. Surface morphology has an effect on reflection and shadowing, influencing the emissivity $\varepsilon$. In this paper, if not specifically indicated, the term emissivity refers to directional spectrum emissivity.

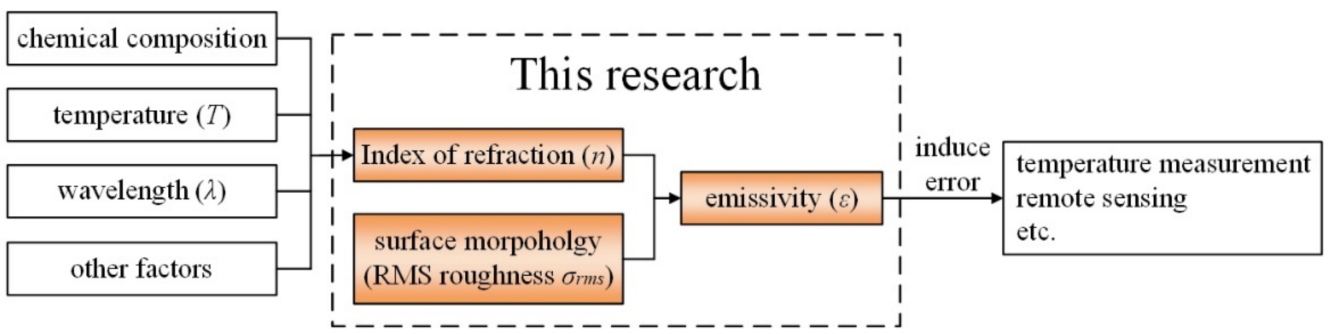

Figure 1. Factors affecting the emissivity $\varepsilon$ of rough surfaces. 
The indirect influences of temperature, wavelength and other factors on emissivity can be experimentally measured and are relatively clear. Studies on $\mathrm{Pt}-10 \mathrm{Rh}$ and $\mathrm{SiC}$ indicate that the emissivity of rough surface increases with the increase of temperature and the decrease of wavelength [6]. Similar phenomena are found in difficult-to-process materials including Inconel 718 and Ti6Al4V [3,7-9]. Other factors including chemical composition [1], heat treatment $[2,3]$ and oxidation $[10,11]$ may also have an effect on emissivity. The influence of chemical composition and heat treatment are combined in the influence of the index of refraction $n$. The oxide layers are neglectable if not specifically oxidized.

The influence of surface morphology is complicated and not yet well understood. It is found that coarsely sandblasted samples, compared with finely sandblasted samples, have higher total hemispherical emissivity at low temperature, but lower emissivity in high temperature [12]. It is also reported that differently processed surfaces with close surface roughness Ra may have very different emissivities [13]. Further information is needed to understand this ambiguity.

Theoretical modelling studies have been carried out to predict the emissivity of rough surfaces. This was predicted considering the shadowing effect [14]. The surface was however assumed as a grey body, which limits its application. The average probability that infrared radiation emitted hits the surface directly was calculated, and the influence of reflection on emissivity of rough surfaces was estimated by Wen and Mudawar [15]. Reflectivity of the rough surface is also affected by viewing angle. Thus, errors were introduced in applying the average probability. Emissivity model of rough surfaces considering shadowing effect and reflection was established [16], while the reflection was assumed no more than once. Emissivity model of sea surfaces have been carried out by Wu and Smith [17] and Li et al. [18-21] respectively. However, sea surfaces have certain patterns (perpendicular to the wind), and are composed merely by sea water. Their models lack in generality.

Despite this valuable work, due to the complexity of the emission processes, the influences of the index of refraction $n$ and surface morphology on the directional emissivity $\varepsilon$ are to be further studied, especially the influence of the index of refraction $n$. In this research, influences of the index of refraction $n$ and root mean square (RMS) roughness $\sigma_{r m s}$ on emissivity $\varepsilon$ are modeled, as shown in Figure 1. Geometric optics are assumed and a directional spectral emissivity prediction model based on the Gaussian random rough surface is briefly analyzed, as similar models have already been proposed and derived in other fields $[18,22]$. Then, a novel matrix method was introduced, and sectional Romberg integral is applied, to numerically solve the proposed model. Influences of the index of refraction $n$ and the root mean square (RMS) roughness $\sigma_{r m s}$ on the directional emissivity $\varepsilon$ are analyzed and discussed. It is found that emissivity of surfaces with lower $\sigma_{r m s}$ and higher $n$ tends to have a peak in high viewing angles. On the contrary, the emissivity of surfaces with higher $\sigma_{r m s}$ and lower $n$ tends to have a peak in low viewing angles. Finally, experimental verification based on IR temperature measurement of an Inconel 718 machined surface is carried out.

\section{Methodology}

In the model part, the surface is assumed to be Gaussian, and limited to the area of geometrical optics (GO). According to the number of reflecting processes involved, the emissivity is divided into components. To contribute to one component, an infrared ray must experience processes including emission, shadowing and reflection. As the surface is random, each process can only be discussed statistically by a possibility density function, and each component calculated by integration. The expression of emissivity is derived by summarizing the components. In this part, it is noticed that the emission, shadowing and reflection processes have no inner connection, and can be expressed separately. Similar processes of different components share the same properties, and can be depicted by one formula. 
The expression of emissivity is too complex to be solved analytically. In the numerical solution part, considering the discoveries above, a matrix method is designed. Emission, shadowing and reflection processes are represented and computed by matrixes. Nested loops are avoided and computational time is shortened.

Experimental verification based on IR temperature measurement of an Inconel 718 machined surface is carried out. Temperature and emissive power are measured simultaneously to calculate the emissivity. Other experimental studies are also cited to further verify the model. The framework of this paper is displayed in Figure 2.

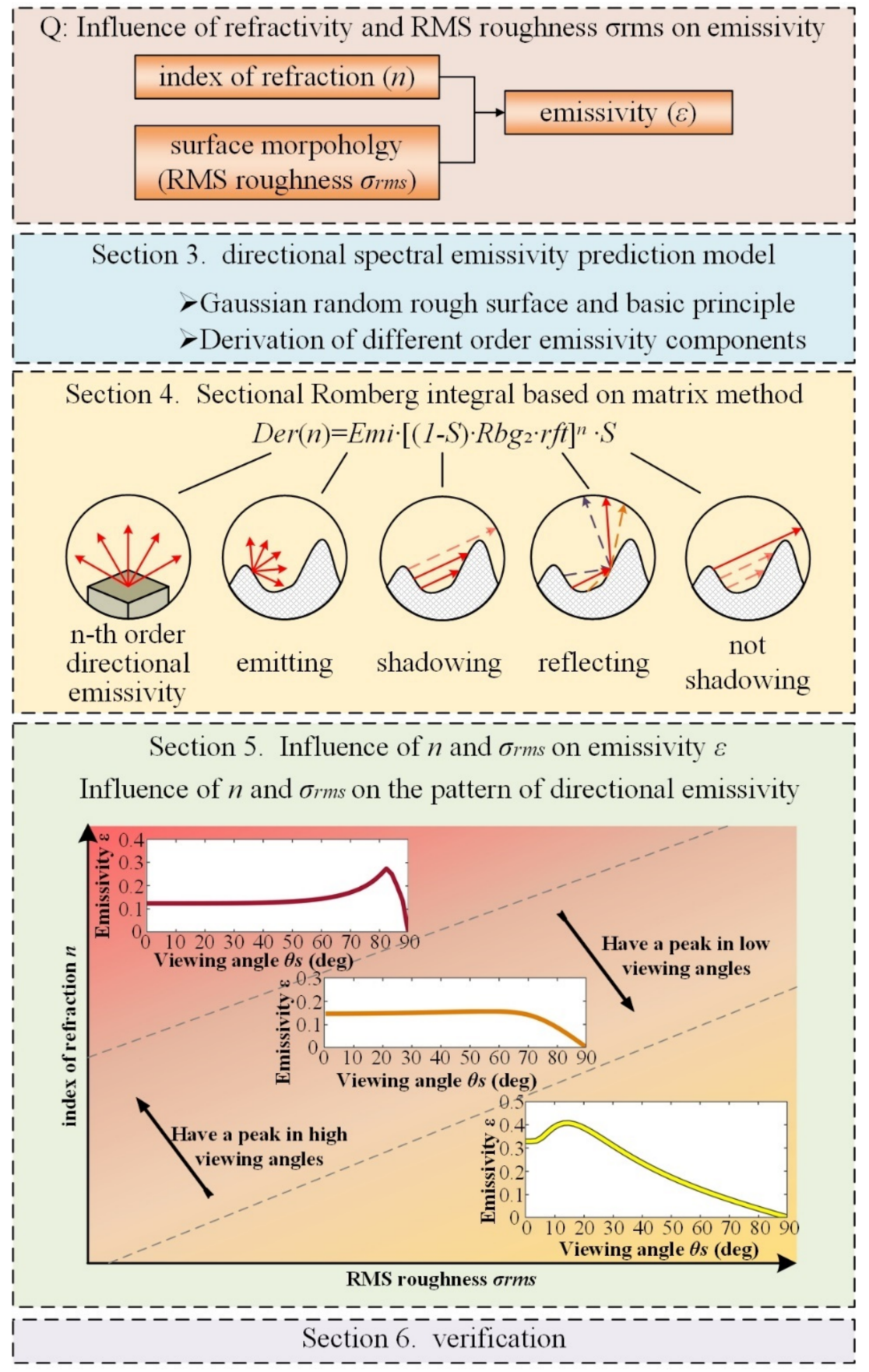

Figure 2. The framework of this paper. 


\section{Modeling of Directional Emissivity of Rough Surfaces}

\subsection{Analysis of Emissivity of Rough Surfaces}

To predict the emissivity of rough surfaces, three-dimensional Gaussian random rough surface is assumed, as shown in Figure 3. The three-dimensional Gaussian random rough surface is generated by a stationary stochastic process, as expressed by Equation (1) [22]:

$$
\begin{gathered}
\langle\zeta(\boldsymbol{r})\rangle=0 \\
\left\langle\zeta(\boldsymbol{r}) \zeta\left(\boldsymbol{r}^{\prime}\right)\right\rangle=\sigma_{r m s}^{2} \cdot e^{\frac{-\left(x_{1}-x_{2}\right)^{2}-\left(y_{1}-y_{2}\right)^{2}}{2 \sigma_{r m s}^{2}}}
\end{gathered}
$$

where $r$ is the position vector, and $x, y, z$ are the coordinates of $r$. We have:

$$
t_{N}\left(p, q, \sigma_{r m s}\right)=\frac{1}{2 \pi \sigma_{r m s}^{2}} \cdot e^{\frac{-p^{2}-q^{2}}{2 \sigma_{r m s}^{2}}}
$$

where $p, q$ are the slopes of the surface in $x, y$ direction, respectively. $t_{N}\left(p, q, \sigma_{r m s}\right)$ is the probability density function of $p, q$. The Gaussian random rough surfaces have the following characteristics:

- The surface is isotropic (and statistically symmetry);

- $\quad p$ and $q$ are randomly distributed according to a Gaussian distribution;

- No correlation between $p$ and $q$.

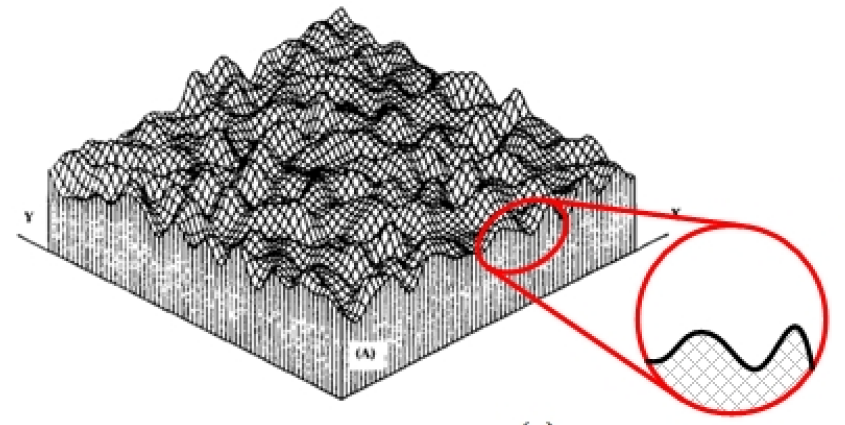

(a)

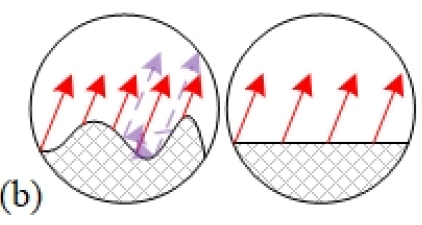

(c)

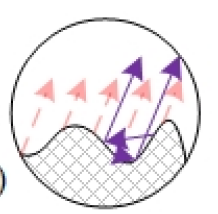

Figure 3. Effect of surface morphology on emissivity. (a): Gaussian random rough surface (Ref. [23], Figure 2) (b): Fresnel's law. (c): Reflection enhancement.

In this study, emissivity is defined as the emissive power of the rough surface compared with a black body, and geometric optics is assumed. According to Fresnel's law and the reflection law:

$$
\varepsilon_{F}\left(n, \theta_{s}\right)= \begin{cases}\frac{\sqrt{n^{2}-\sin ^{2}\left(\theta_{s}\right)}}{n^{2} \cos \left(\theta_{s}\right)+\sqrt{n^{2}-\sin ^{2}\left(\theta_{s}\right)}}+\frac{\cos \left(\theta_{s}\right)}{\cos \left(\theta_{s}\right)+\sqrt{n^{2}-\sin ^{2}\left(\theta_{s}\right)}}, & \tan \theta_{s}<n \\ \frac{n^{2} \cos \left(\theta_{s}\right)}{n^{2} \cos \left(\theta_{s}\right)+\sqrt{n^{2}-\sin ^{2}\left(\theta_{s}\right)}}+\frac{\cos \left(\theta_{s}\right)}{\cos \left(\theta_{s}\right)+\sqrt{n^{2}-\sin ^{2}\left(\theta_{s}\right)}}, & \tan \theta_{s} \geq n\end{cases}
$$

where $\varepsilon_{F}$ is emissivity, $n$ is the index of refraction, $\theta_{S}$ is the viewing angle.

The main phenomena in the emission process of rough surfaces are Fresnel's law and reflection enhancement. Figure $3 b$ shows that the smooth surface consists of many horizontal microelements, while the rough surface consists of elements with varied directions, each having different emissivity in the same direction. Reflection enhancement refers to the phenomenon whereby reflection induces higher emissivity near the normal direction in rough surfaces, as depicted in Figure 3c. Reflection enhancement may also cause lower emissivity in high viewing angles. Due to their combined effect, the influence of surface morphology is complicated and not yet well understood. 


\subsection{Modelling of Emissivity of Rough Surfaces}

Directional spectral emissivity refers to the ability of measured surface to emit in a certain wavelength and direction compared with a black body. A statistical model is established to calculate the emissivity of rough surfaces.

Infrared radiation emitted by an element may leave the surface directly (zeroth-order), reflect once (first-order), twice (second-order), and so on. Considering that the correlation between emissivity and the emitted power is linear, the emissivity could be divided into different order components, and the components can be computed separately. Components of second order and above are called high-order components collectively, as presented in Equation (5):

$$
\varepsilon=\sum_{i=0}^{\infty} \varepsilon_{i}
$$

Zeroth-order component refers to emissivity related to infrared radiation that leaves the surface directly. To contribute to zeroth-order component, an infrared ray must be emitted, and not be blocked by another part of the surface. Different elements have different directions, and thus have different local viewing angle $\theta_{l}$, as illustrated in Figure $4 \mathbf{b}$. According to Equation (4), their local emissivities $\varepsilon_{l}$ are not equal. The emitted radiation ray may also be blocked by another element, and the possibility is a function of its slope.

(a)

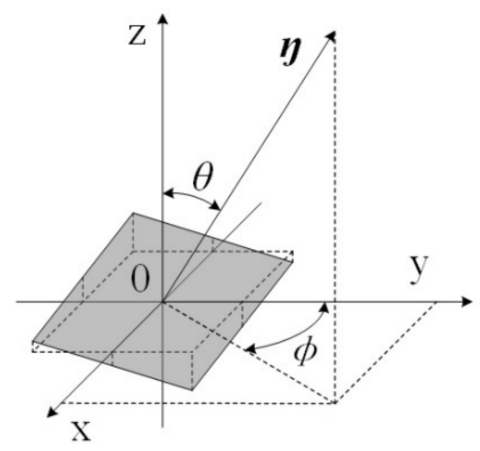

(b)

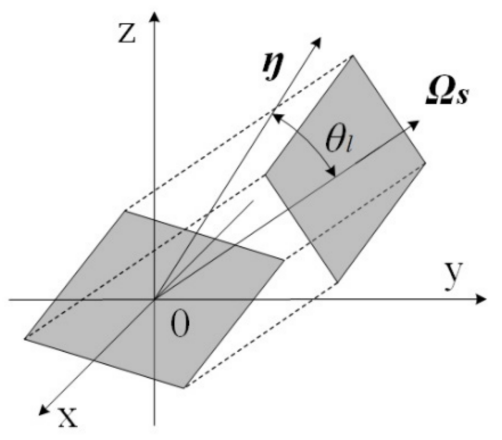

Figure 4. Zeroth-order component (a): Element. (b): Projection of the element.

In Figure 4a:

$$
\eta=\left(\frac{-p}{\sqrt{p^{2}+q^{2}+1}}, \frac{-q}{\sqrt{p^{2}+q^{2}+1}}, \frac{1}{\sqrt{p^{2}+q^{2}+1}}\right)
$$

where $\eta$ is the normal direction of the element. As shown in Figure $4 b$, projection of the element distorts depending on $\eta$ and the viewing direction $\Omega_{S}$. Thus, area projection coefficient $A_{p}$ is:

$$
A_{p}=\left\{\begin{array}{cc}
\boldsymbol{\eta}_{y o z} \cdot \boldsymbol{\Omega}_{s}=\cos \left(\theta_{s}\right)-q \sin \left(\theta_{s}\right), & q<\cot \left(\theta_{s}\right) \\
0, & q \geq \cot \left(\theta_{s}\right)
\end{array}\right.
$$

where $\eta_{y o z}$ is the component of $\eta$ in plane yoz.

$$
t\left(\theta_{s}, p, q, \sigma_{r m s}\right)=\frac{A_{p} \cdot e^{\frac{-p-q}{2 \sigma_{r m s}^{2}}}}{\int_{-\infty}^{\cot \theta_{s}} \int_{-\infty}^{\infty} A_{p} \cdot e^{\frac{-p^{2}-q^{2}}{2 \sigma_{r m s}^{2}}} d p d q}, \quad\left(q<\cot \theta_{s}\right)
$$

where $t\left(\theta_{s}, p, q, \sigma_{r m s}\right)$ is the possibility that the slope of the emitting element is $p, q$. Smith's shadowing function $S\left(\theta_{s}, \sigma_{r m s}\right)[24,25]$ is the possibility that infrared radiation is 
not blocked. It has been reported [26,27] that correlation between elements is not significant and can be ignored. Smith's shadowing function is estimated as Equation (9):

$$
\begin{gathered}
S\left(\theta_{s}, \sigma_{r m s}\right)== \begin{cases}\frac{1}{\Lambda\left(\theta_{s}, \sigma_{r m s}\right)+1}, & \theta_{s}<\frac{\pi}{2} \\
0, & \theta_{s} \geq \frac{\pi}{2}\end{cases} \\
\Lambda\left(\theta_{s}, \sigma_{r m s}\right)=\frac{1}{2} \sqrt{\frac{2}{\pi}} \cdot \frac{\sigma_{r m s}}{\cot \theta_{s}} e^{-\frac{\cot ^{2} \theta_{s}}{2 \sigma_{r m s}}-\operatorname{erfc}\left(\frac{\cot \theta_{s}}{\sqrt{2} \sigma_{r m s}}\right)} \\
\varepsilon_{l}\left(n, p, q, \theta_{s}\right)= \begin{cases}\frac{\sqrt{n^{2}-\sin ^{2}\left(\theta_{s l}\right)}}{n^{2} \cos \left(\theta_{s l}\right)+\sqrt{n^{2}-\sin ^{2}\left(\theta_{s l}\right)}}+\frac{\cos \left(\theta_{s l}\right)}{\cos \left(\theta_{s l}\right)+\sqrt{n^{2}-\sin ^{2}\left(\theta_{s l}\right)}}, & \tan \theta_{s l}<n \\
\frac{n^{2} \cos \left(\theta_{s l}\right)}{n^{2} \cos \left(\theta_{s l}\right)+\sqrt{n^{2}-\sin ^{2}\left(\theta_{s l}\right)}}+\frac{\cos \left(\theta_{s l}\right)}{\cos \left(\theta_{s l}\right)+\sqrt{n^{2}-\sin ^{2}\left(\theta_{s l}\right)}}, & \tan \theta_{s l} \geq n\end{cases} \\
\theta_{s l}\left(\theta_{s}, p, q\right)=\cos ^{-1}\left(\frac{\cos \left(\theta_{s}\right)-q \sin \left(\theta_{s}\right)}{\sqrt{1+p^{2}+q^{2}}}\right)
\end{gathered}
$$

The zeroth-order component is,

$$
\varepsilon_{0}=\int_{-\infty}^{\cot \theta_{s}} \int_{-\infty}^{\infty} t\left(\theta_{s}, p, q, \sigma_{r m s}\right) S\left(\theta_{s}, \sigma_{r m s}\right) \varepsilon_{l}\left(n, p, q, \theta_{s}\right) d p d q
$$

In Equation (13), we note that $S\left(\theta_{s}, \sigma_{r m s}\right)$ is not a function of $p, q$. The integration part depicts the emission process, while $S\left(\theta_{s}, \sigma_{r m s}\right)$ stands for not being blocked.

The first-order component refers to emissivity related to infrared radiation that reflects once before leaving the surface. To contribute to a zeroth-order component, an infrared ray must be emitted, hit another part of the surface, be reflected, and not hit the surface again. The process could be divided into two parts: emission and blocking, reflecion and exiting. Figure 5 shows that the infrared radiation emitted by the first element hits the second element in direction $\Omega_{1}$, and the reflected beam leaves the surface in direction $\Omega_{S}$. By applying the local coordinate system $x^{\prime} y^{\prime} z^{\prime}$, the emission process is similar to the zeroth-order component.

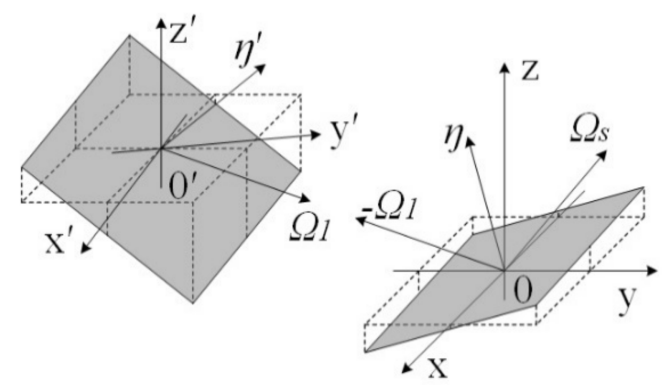

Figure 5. First-order component.

According to the reflection law [28]:

$$
\eta=\frac{\Omega_{s}-\Omega_{1}}{\left|\Omega_{s}-\Omega_{1}\right|}
$$

which is:

$$
\begin{gathered}
p=\frac{\sin \theta_{1} \sin \phi_{1}}{\cos \theta_{s}-\cos \theta_{1}} \\
q=\frac{\sin \theta_{1} \cos \phi_{1}-\sin \theta_{s}}{\cos \theta_{s}-\cos \theta_{1}}
\end{gathered}
$$


By substituting Equations (11) and (12) into Equations (7) and (8), $t\left(\theta_{1}, p, q, \sigma_{r m s}\right)$ and $\varepsilon_{l}\left(n, p, q, \theta_{s}\right)$ could be expressed as $t\left(\theta_{s}, \theta_{1}, \phi_{1}, \sigma_{r m s}\right)$ and $\varepsilon_{l}\left(n, \theta_{1}, \phi_{1}, \theta_{s}\right)$. According to Kirchhoff's law we have [28]:

$$
\rho=1-\alpha=1-\varepsilon_{l}
$$

where $\alpha, \rho$ are the absorptivity and reflectivity, respectively. Noting that Gaussian random rough surface is isotropic and statistically symmetry, we have:

$$
\begin{aligned}
& \varepsilon_{1}=\int_{0}^{2 \pi} \int_{0}^{\pi-\theta_{s}} i\left(\theta_{1}, \sigma_{r m s}\right) t\left(\theta_{s}, \theta_{1}, \phi_{1}, \sigma_{r m s}\right) S\left(\theta_{s}, \sigma_{r m s}\right)\left[1-\varepsilon_{l}\left(n, \theta_{1}, \phi_{1}, \theta_{s}\right)\right] \cdot \sin \theta_{1} d \theta_{1} d \phi_{1} \\
& i\left(\theta_{1}, \sigma_{r m s}\right)=\int_{-\infty}^{\cot \theta_{1}} \int_{-\infty}^{\infty} t\left(\theta_{1}, p_{1}, q_{1}, \sigma_{r m s}\right)\left[1-S\left(\theta_{1}, \sigma_{r m s}\right)\right] \varepsilon_{l}\left(n, \theta_{1}, \phi_{1}, \theta_{s}\right) d p_{1} d q_{1}
\end{aligned}
$$

where Equation (18) refers to the reflection process, and Equation (19) refers to the emitting process. Noting that $S\left(\theta_{s}, \sigma_{r m s}\right)$ is not a function of $p, q$. Equation (13b) is mainly about emission and blocking, while Equation (18) is about reflection and exiting. In Equation (19), the integration part depicts the emission process, while $\left[1-S\left(\theta_{s}, \sigma_{r m s}\right)\right]$ stands for blocking, which is similar to Equation (13). In Equation (19), $S\left(\theta_{s}, \sigma_{r m s}\right)$ stands for exiting, and the rest for reflection.

As shown in Figure 6, second-order component refers to emissivity related to infrared radiation that reflects twice before leaving the surface. By applying a local coordinate system to the first element and the second element, we have:

$$
\begin{aligned}
& \varepsilon_{2}=\int_{0}^{2 \pi} \int_{0}^{\pi-\theta_{s}} i_{2}\left(\theta_{2}, \sigma_{r m s}\right) t\left(\theta_{s}, \theta_{2}, \phi_{2}, \sigma_{r m s}\right) S\left(\theta_{s}, \sigma_{r m s}\right)\left[1-\varepsilon_{l}\left(n, \theta_{2}, \phi_{1}\right)\right] \cdot \sin \theta_{2} d \theta_{2} d \phi_{2} \\
& i_{2}\left(\theta_{2}, \sigma_{r m s}\right)=\int_{-\infty}^{\cot \theta_{2}} \quad \int_{-\infty}^{\infty} i_{1}\left(\theta_{1}, \sigma_{r m s}\right) t\left(\theta_{s}, \theta_{1}, \phi_{1}, \sigma_{r m s}\right) \\
& {\left[1-S\left(\theta_{s}, \sigma_{r m s}\right)\right]\left[1-\varepsilon_{l}\left(n, \theta_{1}, \phi_{1}\right)\right] \cdot \sin \theta_{1} d \theta_{1} d \phi_{1}} \\
& i_{1}\left(\theta_{1}, \sigma_{r m s}\right)=\int_{-\infty}^{\cot \theta_{1}} \int_{-\infty}^{\infty} t\left(\theta_{1}, p_{1}, q_{1}, \sigma_{r m s}\right)\left[1-s\left(\theta_{1}, \sigma_{r m s}\right)\right] \varepsilon_{l}\left(n, p_{1}, q_{1}\right) d p_{1} d q_{1}
\end{aligned}
$$

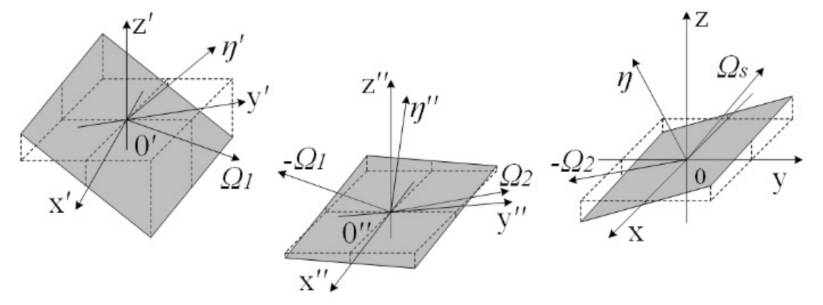

Figure 6. Second-order component.

Third order component and above are modeled similarly. We note that emissivity is not a function of $\varphi$ and the model is symmetrical.

\section{Numerical Solution Based on a Matrix Method}

The directional spectral emissivity of the rough surfaces is too complex to be solved analytically. Generally, multiple integrals are computed by nested loops, and the operation period of nested loops increases exponentially with multiplicity. In this section, a novel matrix method is introduced, and sectional Romberg integral is applied to obtain numerical solutions quickly and precisely.

As shown in Section 3.2, high-order components are related to two basic process, emission and refraction. As mentioned in Section 3.1, a Gaussian random rough surface is isotropic (and symmetrical), and no correlation between $p$ and $q$ is assumed. From another point of view, the optics processes involved are emission, reflection and shadowing. Thus, 
these processes can be represented by irrelevant matrixes. The numerical integration could be obtained by multiplying matrixes, which is much quicker than nested loops. We define:

$$
\operatorname{Der}(n)=E m i \cdot\left[(1-S) \cdot R b g_{2} \cdot r f t\right]^{n}
$$

where $\operatorname{Der}(n)$ is the $n$-th order emissivity in the viewing angle $\theta_{s_{k}}$. Emi is the emission matrix. $S$ is the shadowing matrix. $R b g_{2}$ is the Romberg matrix. Definitions of $R b g_{2}$ and theoretical verification of the matrix method are presented in Appendix A. rft is the reflection matrix. This gives:

$$
\begin{gathered}
E m i=\left[\begin{array}{lllll}
e m i_{1} & e m i_{2} & e m i_{3} & \ldots & e m i_{97}
\end{array}\right] \\
e m i_{k}=\int_{-\infty}^{\cot \theta_{s_{k}}} \int_{-\infty}^{\infty} t\left(\theta_{s_{k}}, p, q, \sigma_{r m s}\right) \varepsilon_{F}(n, p, q) d p d q
\end{gathered}
$$

where $\theta_{s_{k}}$ are, respectively.

$$
\begin{gathered}
S=\left[S\left(\theta_{s_{1}}, \sigma_{r m s}\right) S\left(\theta_{s_{2}}, \sigma_{r m s}\right) S\left(\theta_{3}, \sigma_{r m s}\right) \ldots S\left(\theta_{s_{97}}, \sigma_{r m s}\right)\right] \\
r f t=\left[\begin{array}{cccc}
r f t_{11} & r f t_{12} & \ldots & r f t_{197} \\
r f t_{21} & r f t_{22} & \ldots & r f t_{2} 97 \\
\vdots & \vdots & \ddots & \vdots \\
r f t_{971} & r f t_{972} & \ldots & r f t_{9797}
\end{array}\right] \\
r f t_{l k}=\left(\pi-\theta_{s_{k}}\right) \cdot \int_{0}^{2 \pi} t\left(\theta_{s_{k}}, \theta_{l}, \phi, \sigma_{r m s}\right)\left[1-\varepsilon_{l}\left(n, \theta_{l}, \phi\right)\right] \cdot \sin \theta_{l} d \phi
\end{gathered}
$$

From the viewpoint of optics, as sketched in Figure 7, Emi, $(1-S), R b g_{2}, r f t$ are defined, representing the emission, shadowing, reflection and leaving processes, respectively. $\mathrm{emi}_{k}$ is the average emissivity of a random element in direction $\theta_{K}$. Elements of $(1-S)$ are the possibility that a ray in direction $\theta_{K}$ hits the surface again. $R b g_{2}$ is a constant matrix introduced by Romberg integration. $R f t_{l k}$ is the average energy reflected to direction $\theta_{K}$ when a ray from direction $\theta_{l}$ hits a random element. Thus, the infrared rays are traced statistically by a matrix method.

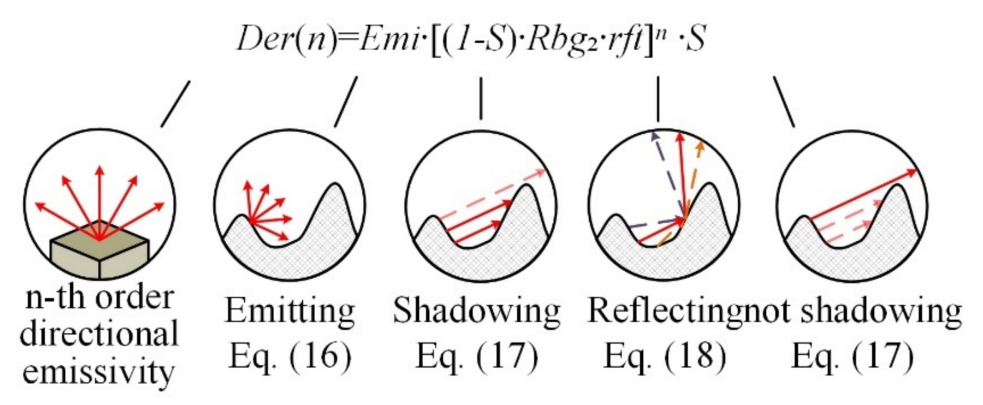

Figure 7. Matrix method, in the viewpoint of optics.

Multiple integrations are generally computed by nested loops, in which $t\left(\theta_{S}, p, q\right.$, $\left.\sigma_{r m s}\right)$ and $\varepsilon_{F}(n, p, q)$ must be calculated repeatedly in every step of every integration. Thus, computational time increases exponentially with the order $n$. In matrix method, they are computed only in obtaining the matrixes. Therefore, the computational time hardly increases when $n>0$. The amount of calculation is presented in Table 1 . Table 2 illustrates that the estimated error of the matrix method is acceptable. Estimation of the error is summarized in Appendix A. 
Table 1. How many times $t\left(\theta_{S}, p, q, \sigma_{r m s}\right)$ and $\varepsilon_{F}(n, p, q)$ are calculated in nested loops and matrix method.

\begin{tabular}{cccccc}
\hline Approach & $\varepsilon_{\mathbf{0}}$ & $\varepsilon_{\mathbf{1}}$ & $\varepsilon_{\mathbf{2}}$ & $\varepsilon_{\mathbf{3}}$ & $\varepsilon_{\mathbf{4}}$ \\
\hline nested loops & $2.62 \cdot 10^{5}$ a & $3.82 \cdot 10^{9}$ & $8.00 \cdot 10^{12}$ & $1.64 \cdot 10^{16}$ & $3.36 \cdot 10^{19}$ \\
matrix method & $2.62 \cdot 10^{5}$ & $4.77 \cdot 10^{8}$ & $4.77 \cdot 10^{8}$ & $4.77 \cdot 10^{8}$ & $4.77 \cdot 10^{8}$ \\
\hline
\end{tabular}

a The results are counted by MATLAB program, when $h_{\theta}=1 / 128 \approx 0.008, h_{p}=\pi / 32 \approx 0.1, \theta_{S}=20^{\circ}, \sigma_{r m s}=0.33$.

Table 2. The influence of $n$ and $\sigma_{r m s}$ on estimated error.

\begin{tabular}{cccccc}
\hline \multirow{2}{*}{ Index of Refraction $\boldsymbol{n}$} & \multicolumn{5}{c}{ RMS Roughness $\sigma_{r m s}$} \\
\cline { 2 - 6 } & $\mathbf{0}$ & $\mathbf{0 . 2}$ & $\mathbf{0 . 5}$ & $\mathbf{1}$ & $\mathbf{2}$ \\
\hline 1 & $2 \cdot 10^{-9} \mathrm{a}$ & $3.55 \cdot 10^{-5}$ & $8.00 \cdot 10^{-5}$ & $6.23 \cdot 10^{-6}$ & $5.65 \cdot 10^{-6}$ \\
2 & $3.18 \cdot 10^{-6}$ & $1.22 \cdot 10^{-4}$ & $2.33 \cdot 10^{-4}$ & $1.80 \cdot 10^{-4}$ & $2.78 \cdot 10^{-4}$ \\
4 & $1.86 \cdot 10^{-6}$ & $2.43 \cdot 10^{-4}$ & $1.65 \cdot 10^{-4}$ & $1.15 \cdot 10^{-4}$ & $1.21 \cdot 10^{-4}$ \\
8 & $1.38 \cdot 10^{-6}$ & $4.13 \cdot 10^{-4}$ & $1.80 \cdot 10^{-4}$ & $2.10 \cdot 10^{-4}$ & $1.26 \cdot 10^{-4}$ \\
16 & $4.59 \cdot 10^{-6}$ & $2.75 \cdot 10^{-4}$ & $3.10 \cdot 10^{-4}$ & $3.54 \cdot 10^{-4}$ & $3.34 \cdot 10^{-4}$ \\
\hline
\end{tabular}

a Accuracy of the PC in this research is $2^{-30}$ (about $10^{-9}$ ). Thus, this data has only one significant figure.

\section{Results and Discussion}

The effects of the index of refraction $n$ and the RMS roughness $\sigma_{r m s}$ on the emissivity $\varepsilon$ are discussed by setting different parameters. The numerical integral was carried out with the MATLAB R2018a program, using a personal computer running with an Intel Core $\mathrm{I7}-4720 \mathrm{HQ}, 3.6 \mathrm{GHz}$. CPU. In industry, $n$ is mostly between 2 and $16, \sigma_{r m s}$ is mostly under 1 . The values of $n$ and $\sigma_{r m s}$ is chosen inside this range by proportional sequences, to cover the majority of rough surfaces encountered in industry.

\subsection{Pattern of Different Components}

The patterns of different components are discussed. Figure 8 displays that higher order components are considerably smaller in scale. The proportion of higher order components increases with increasing $\sigma_{r m s}$. The components of first order and above are almost 0 when the surface is smooth, and consists of only about $20 \%$ when the surface is relatively rough.

\subsection{Effects of the Index of Refraction $n$ on Emissivity $\varepsilon$}

The index of refraction $n$ depends on the chemical composition of the surface and varies with wavelength and temperature [28].

When the RMS roughness $\sigma_{r m s}$ is relatively high, $\varepsilon$ would be dominated by $\sigma_{r m s}$, which is to be discussed in Section 5.3. When $\sigma_{r m s}$ is relatively low, $p, q$ converge to zero, and the surface would be too smooth for an infrared ray emitted to hit the surface again. Thus, $\varepsilon$ depends mainly on $\varepsilon_{0}$, and $\varepsilon_{l} \approx \varepsilon_{F}$. Equation (6) could be transformed into the following form:

$$
\begin{gathered}
\varepsilon_{0}=S\left(\theta_{s}, \sigma_{r m s}\right) \operatorname{emi}\left(\theta_{s}, n, \sigma_{r m s}\right) \\
\operatorname{emi}\left(\theta_{s}, n, \sigma_{r m s}\right)=\int_{-\infty}^{\cot \theta_{s}} \int_{-\infty}^{\infty} t\left(\theta_{s}, p, q, \sigma_{r m s}\right) \varepsilon_{l}\left(n, p, q, \theta_{s}\right) d p d q
\end{gathered}
$$

The patterns shown in Figure $9 \mathrm{~b}$ are reported in experimental studies [14,29]. It is shown in Figure 9 that $\varepsilon_{F}$ and $\varepsilon$ have similar patterns. Obviously, $n$ affects $\varepsilon_{l}\left(\varepsilon_{l} \approx \varepsilon_{F}\right)$, and thus have an effect on $\varepsilon$. In another word, $n$ influences $\varepsilon_{l}$ by influencing the emission characteristics of the elements. emi $\left(\theta_{S}, n, \sigma_{r m s}\right)$ is the same function that stands for the emission process mentioned in Section 4. In Equation (4), it can be analytically demonstrated that the extreme point is the polarizing angle, which is really interesting. Such a phenomenon could be valuable for further research. 


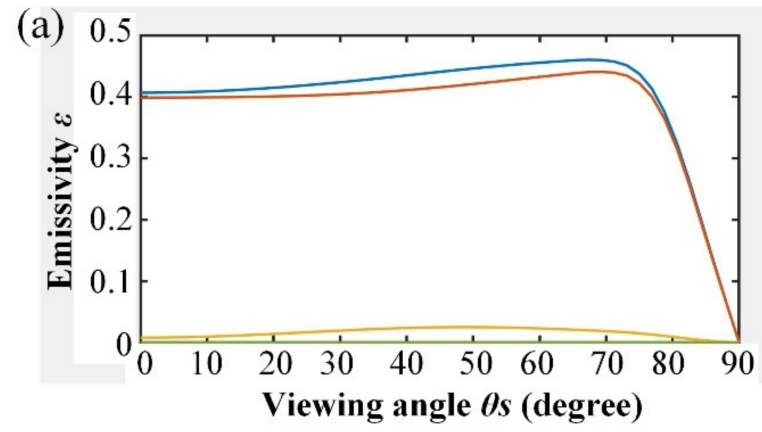

\section{- directional \\ - 0-th order component \\ - 1st order component \\ - 2nd order component \\ - 3rd order component}

(b)

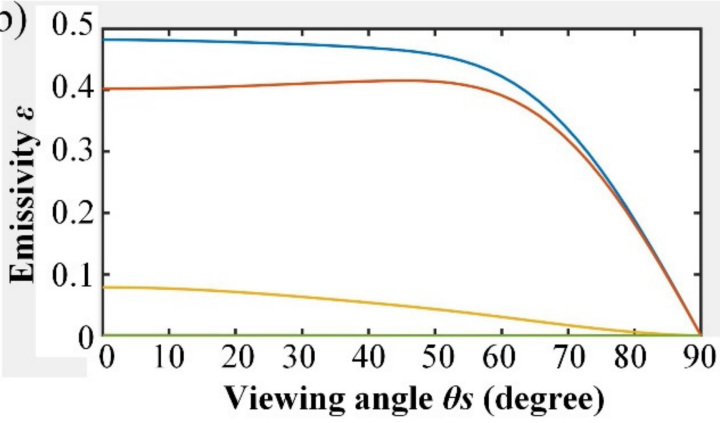

- directional

- 0-th order component

- 1st order component

- 2nd order component

- 3rd order component

(c)

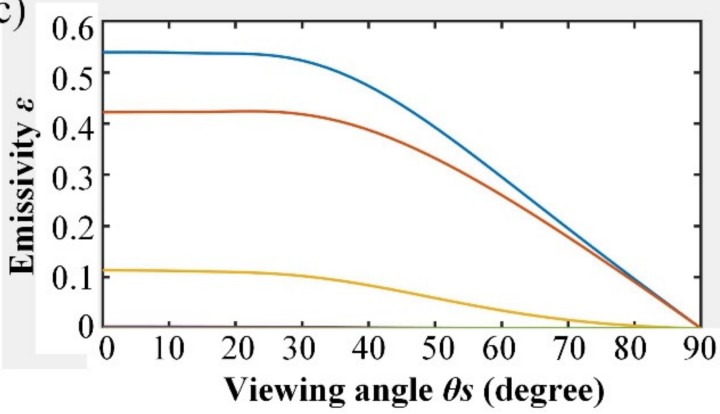

- directional

- 0-th order component

- 1st order component

- 2nd order component

- 3rd order component

Figure 8. Components of emissivity (a): $n=4, \sigma_{r m s}=0.3 ;(\mathbf{b}): n=4, \sigma_{r m s}=0.5 ;(\mathbf{c}): n=4, \sigma_{r m s}=1$.

(a)

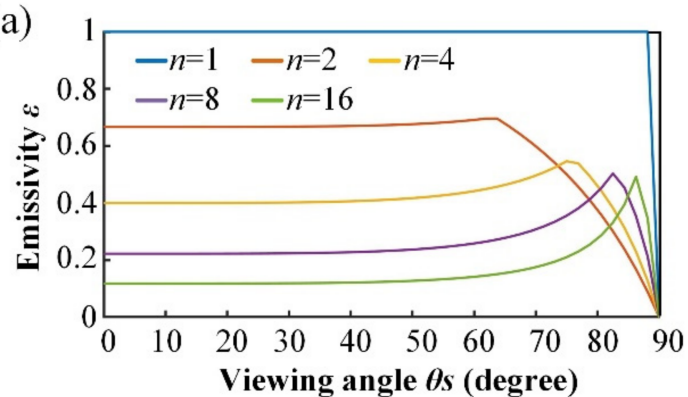

(b)

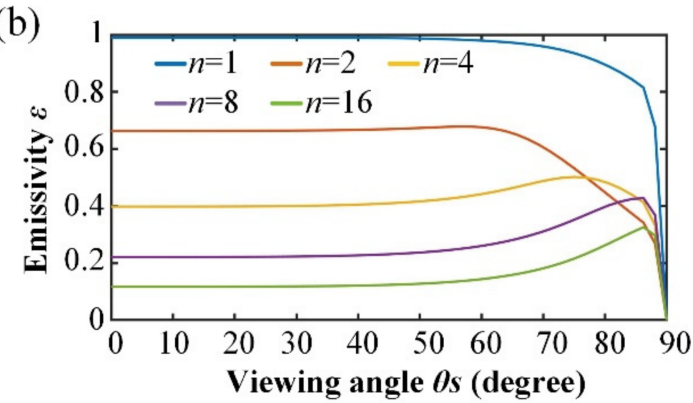

Figure 9. Comparison of the emissivity of elements and surfaces $\left(\sigma_{r m s}=0.1\right)$. (a): Effect of the index of refraction $n$ on emissivity of the elements $\varepsilon_{F}$. (b): Effect of $n$ on emissivity of the surface $\varepsilon$.

\subsection{Effects of RMS Roughness $\sigma_{r m s}$ on Emissivity $\varepsilon$}

Referring to the root mean square of the slope of the surface elements, RMS roughness $\sigma_{r m s}$ is a surface integrity parameter. According to Equations (29) and (30), $\sigma_{r m s}$ affects both $S\left(\theta_{S}, \sigma_{r m s}\right)$ and emi $\left(\theta_{S}, n, \sigma_{r m s}\right)$. That is, $\sigma_{r m s}$ effects both of emission and shadowing. $\varepsilon$ decreases with increasing $\sigma_{r m s}$ in high viewing angle, increases with increasing $\sigma_{r m s}$ in middle viewing angle, and first increases then decreases with increasing $\sigma_{r m s}$ in low viewing angle (near normal direction). Surfaces with very high $\sigma_{r m s}$ may have a peak in 
low viewing angle. It should be noted that high, middle and low here are a relative concept. As higher order components are relatively small, reflection is not discussed in details here.

As shown in Figure 10, on the one hand, the shadowing effect becomes more notable with increasing $\sigma_{r m s}$. Figure 10a shows that $S\left(\theta_{S}, \sigma_{r m s}\right)$ decreases with increasing $\sigma_{r m s}$ in high viewing angle. Thus, $\varepsilon$ decreases with increasing $\sigma_{r m s}$ in high viewing angle. On the other hand, these blocked infrared rays are redistributed in all directions, including middle viewing angles. Thus, $\varepsilon$ increases with increasing $\sigma_{r m s}$ in middle viewing angle.

(a)

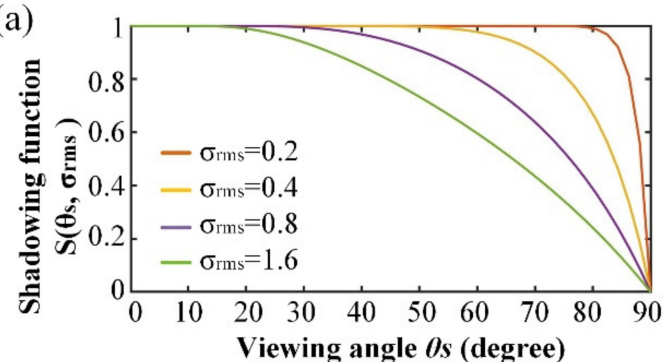

(c)

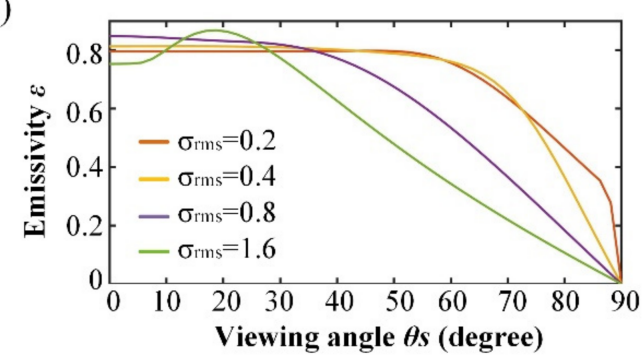

(b)

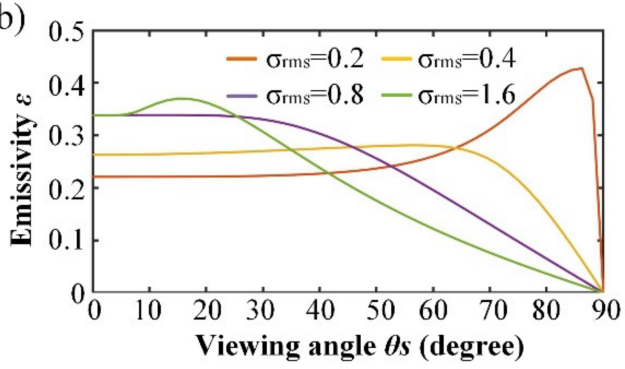

Figure 10. Effect of RMS roughness $\sigma_{r m s}$ on emitting process. (a): Effect on shadowing function $S\left(\theta_{S}\right.$, $\left.\sigma_{r m s}\right)$. (b): Effect on $\varepsilon$ when $n=8$. (c): Effect on $\varepsilon$ when $n=1.5$.

On the other hand, emi $\left(\theta_{S}, n, \sigma_{r m s}\right)$ varies with increasing $\sigma_{r m s}$. Local emissivity of the elements $\varepsilon_{F}\left(\theta_{S}, n\right)$ are not a function of $\sigma_{r m s}$, but the distribution of the elements can be influenced by $\sigma_{r m s}$. Thus, $\sigma_{r m s}$ have an effect on the emissivity $\varepsilon$. As shown in Figure 11, emi $\left(\theta_{S}, n, \sigma_{r m s}\right)$ could be considered as a convolution of $\varepsilon_{F}\left(\theta_{S}, n\right)$. Thus, the curve is flattened and the emissivity decreases with increasing $\sigma_{r m s}$ in high viewing angles, and increases with increasing $\sigma_{r m s}$ in middle viewing angles. The emissivity $\varepsilon$ is dominated by $\sigma_{r m s}$, and $n$ have little effect on $\varepsilon$, as mentioned in Section 5.2. Similar patterns are reported experimentally $[13,20]$.
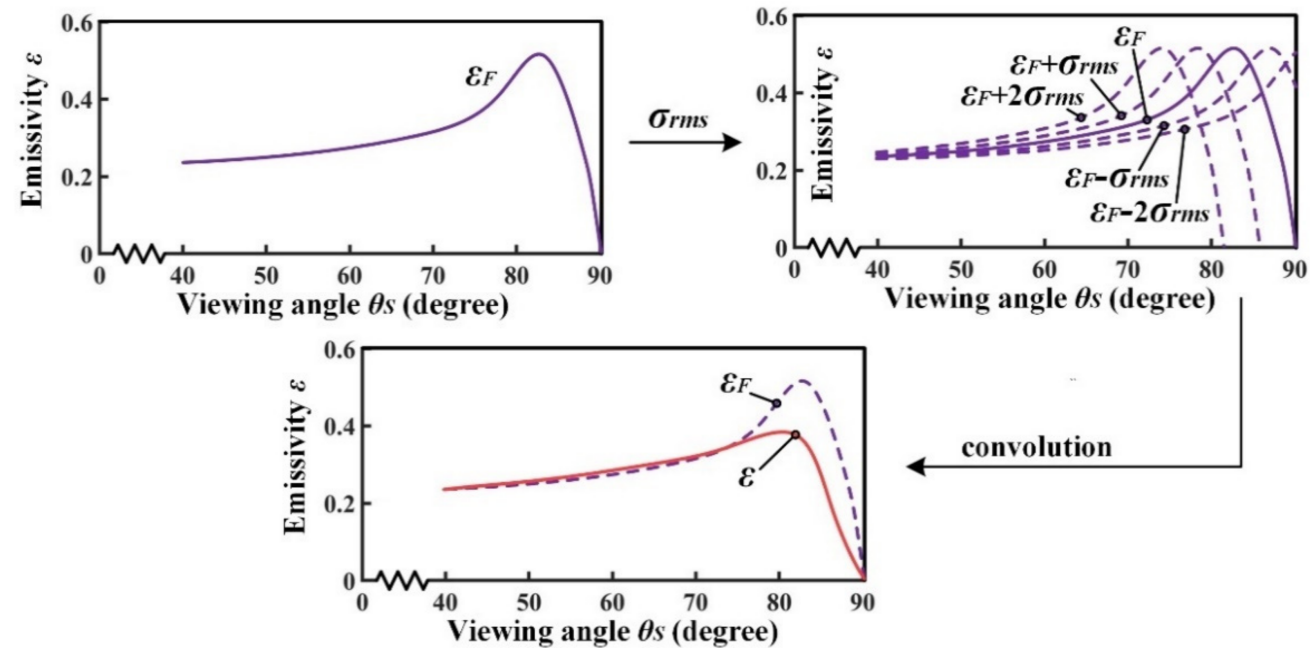

convolution

Figure 11. Effect of RMS roughness $\sigma_{r m s}$ on emitting process and emissivity $\varepsilon$. 
Near normal direction, the effect of $\sigma_{r m s}$ in middle viewing angle still works, and $\varepsilon$ increase with increasing $\sigma_{r m s}$ firstly. While $\sigma_{r m s}$ is very high, $2 \cdot \sigma_{r m s}$ may proceed the polarizing angle, where $\varepsilon_{F}$ starts to decrease with increasing $\theta_{S}$. Thus, $\varepsilon$ near normal direction may decrease with increasing $\sigma_{r m s}$. Due to this phenomenon near normal direction, surfaces with very high $\sigma_{r m s}$ tend to have a peak in low viewing angles.

\subsection{Combined Effect of $n$ and $\sigma_{r m s}$ on $\varepsilon$}

Influences of the index of refraction $n$ and root mean square (RMS) roughness $\sigma_{r m s}$ on emissivity $\varepsilon$ are discussed based on Sections 5.2 and 5.3. $n$ have an impact on emi $\left(\theta_{S}, n\right.$, $\left.\sigma_{r m s}\right)$ by $\varepsilon_{F}$, and thus influences $\varepsilon$. $\sigma_{r m s}$ influences both $e m i\left(\theta_{S}, n, \sigma_{r m s}\right)$ and $S\left(\theta_{S}, \sigma_{r m s}\right)$. In optics point of view, $n$ influences primarily emission process, while $\sigma_{r m s}$ influences both emission and shadowing, as shown in Figure 12.

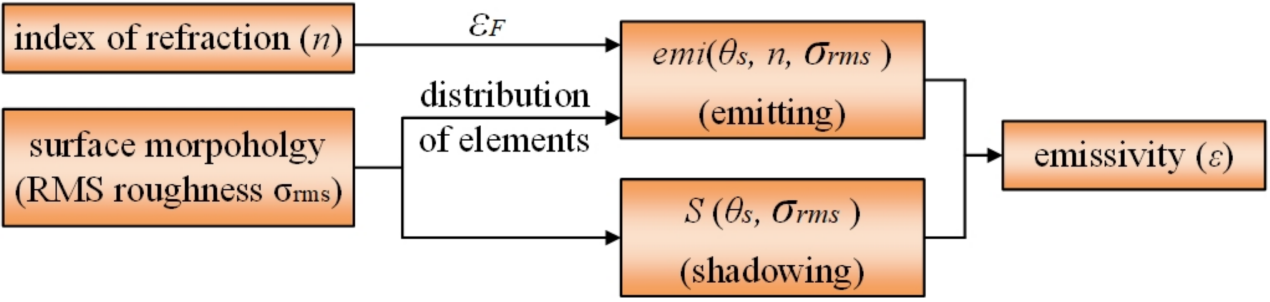

Figure 12. Mechanism of the influence of the index of refraction $n$ and RMS roughness $\sigma_{r m s}$ on emissivity $\varepsilon$.

When $\sigma_{r m s}$ is relatively high, $\varepsilon$ is dominated by $\sigma_{r m s}$. It is found that $\varepsilon$ decreases with increasing $\sigma_{r m s}$ in high viewing angle, increases with increasing $\sigma_{r m s}$ in middle viewing angle, and first increases then decreases with increasing $\sigma_{r m s}$ near normal direction. Therefore, surfaces with very high $\sigma_{r m s}$ may have a peak in low viewing angle. When $\sigma_{r m s}$ is relatively low, $\varepsilon$ tend to decrease with increasing $n$, and have a steeper peak in high viewing angles. The extreme point is the Brewster's angle, which increases with increasing $n$.

Summarizing the conclusions above, surfaces can be divided into three areas depending on index of refraction $n$ and RMS roughness $\sigma_{r m s}$. Surfaces with lower $\sigma_{r m s}$ and higher $n$ tends to have a peak in high viewing angles [30-32]. On the contrary, surfaces with higher $\sigma_{r m s}$ and lower $n$ tends to have a peak in low viewing angles. There is an intermediate region between the two areas [29,32], as shown in Figure 13.

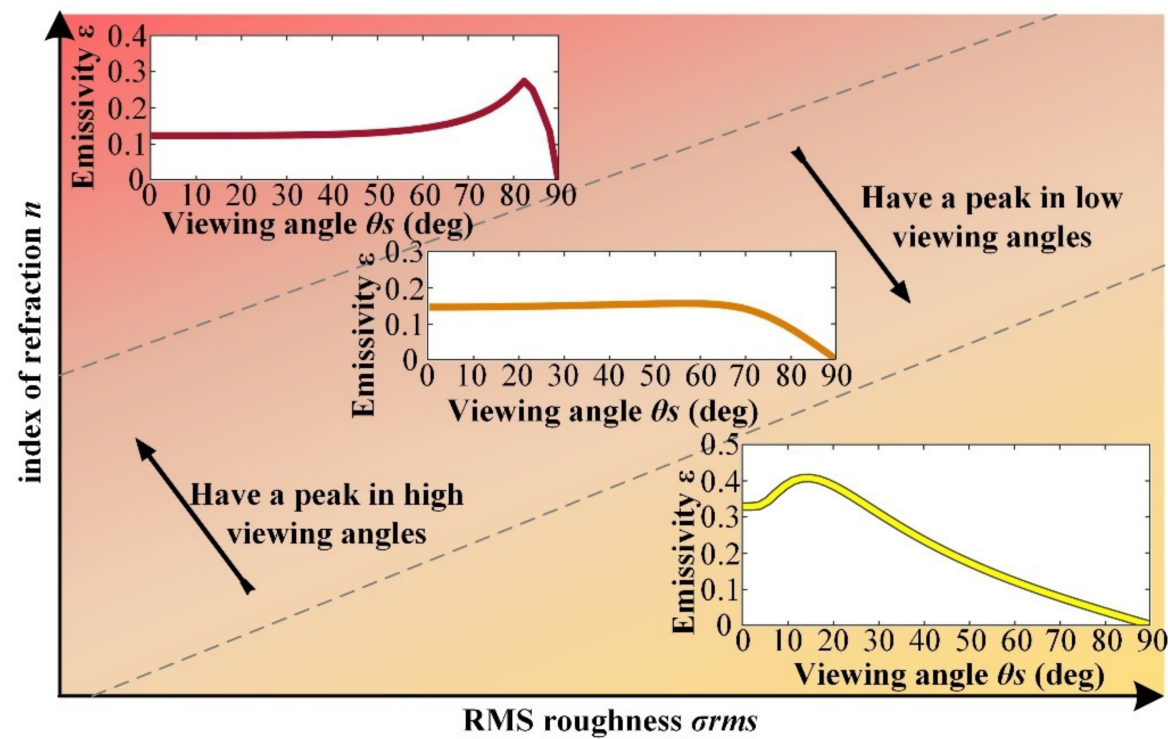

Figure 13. Influence of the index of refraction $n$ and RMS roughness $\sigma_{r m s}$ on the pattern of emissivity. 


\section{Verification by Simulated Experiment}

According to Plunk's law:

$$
E(\lambda, T)=\varepsilon \frac{C_{1} \lambda^{-5}}{e^{\frac{C_{2}}{\lambda T}}-1}
$$

where $C_{1} \approx 3.7418 \cdot 10^{-16} \mathrm{~W} \cdot \mathrm{m}^{2}, C_{2} \approx 1.4388 \cdot 10^{-2} \mathrm{~m} \cdot \mathrm{K}$.

Thus, the emissivity of the surface could be calculated by measuring the temperature $T$ and the emissive power $E(\lambda, T)$. Figure 14a depicts the principle of the proposed verification experiment. The temperature $T$ of the sample is measured by thermocouples. The emissive powe $E(\lambda, T)$ is measured by an IR thermometer from different directions. All data is imported to a personal computer, by which the emissivity $\varepsilon$ is calculated according to Equation (31). All samples are heated to one same temperature (900 K), in order to minimize the error.
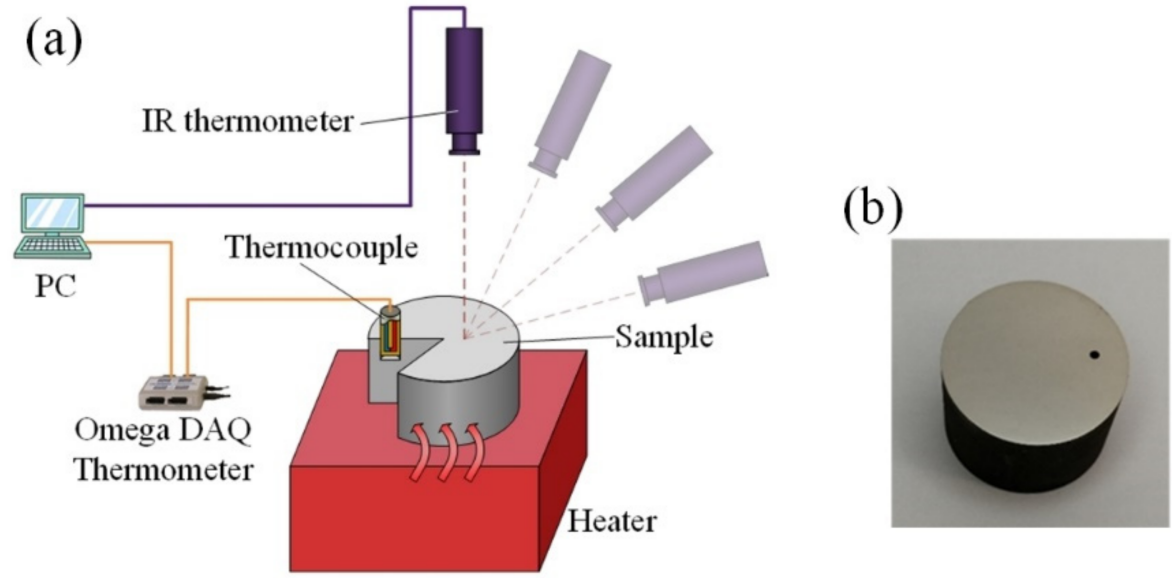

(c)

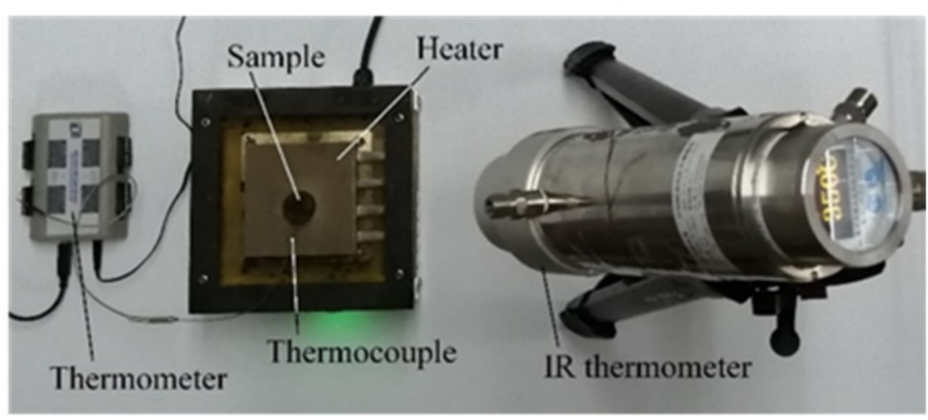

Figure 14. Experimental setup of IR temperature measurement. (a): Principle of the measurement. (b): The sample in Figure 14a. (c): The equipment and sample involved in Figure 14a.

As shown in Figure 14b, the samples introduced are polished Inconel 718 columns, $\phi 27 \times 18 \mathrm{~mm}$, with a blind hole drilled to place the thermocouple. Inconel 718 is a difficultto-process material. The surface roughness of the samples were Ra $0.17 \pm 0.009 \mu \mathrm{m}$, and the RMS roughness were $1.16 \pm 0.05$. State and properties of the sample were referred in [33]. As shown in Figure 14c, the temperature values of the samples were measured by a DAQ-2401 thermometer (OMEGA, city, state abbrev if USA, country) equipped with a type-K thermocouple.

The emissive power $E(\lambda, T)$ was measured by the IR thermometer. The wavelength of the IR thermometer is $1.7 \mu \mathrm{m}$. Further information was reported by Zhao [34] et al. The upper surface of the sample was heated to $900 \mathrm{~K}$ to minimum the environmental interference. Five points in one sample are chosen for each group in the experimental study. As shown in Figure 15, the experimental results match well with the model. 


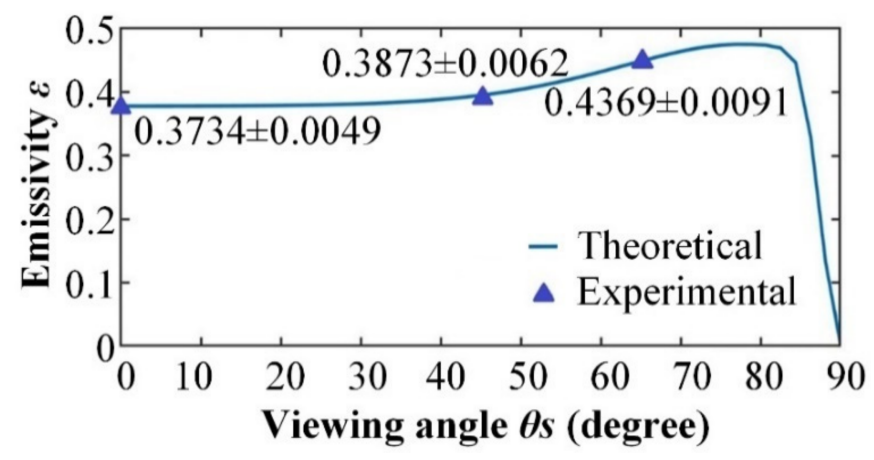

Figure 15. Comparison between experimental and theoretical results (index of refraction $n=4.34$ ).

Sandblasted surfaces have similar pattern with Gaussian random rough surface. Emissivity of Inconel 718 sandblasted surfaces were reported by Campo [35] et al. Figure 16 shows that the experimental results match well with the theoretical results. Deviations at higher viewing angles might due to diffraction.

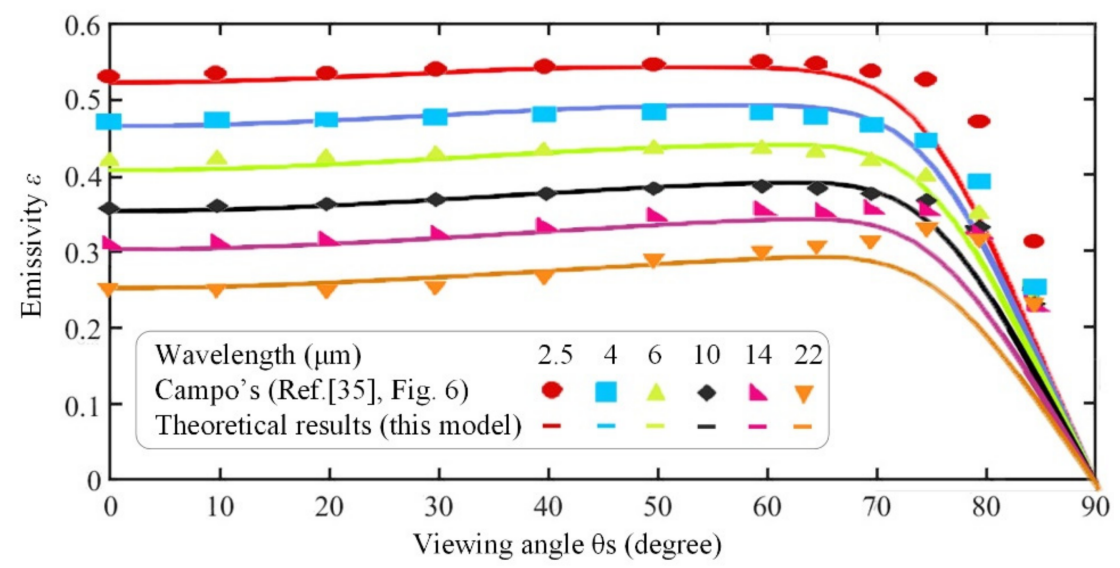

Figure 16. Experimental and theoretical results of smooth surface (Where RMS roughness $\sigma_{r m s}=0.35$, index of refraction $n=2.85,3.35,4.0,4.8,5.8,7.2$, respectively).

\section{Conclusions}

In this research, a theoretical model considering the index of refraction $n$ and the root mean square (RMS) roughness $\sigma_{r m s}$ is established out to predict the emissivity $\varepsilon$ of rough surfaces. The directional spectral emissivity prediction model based on the Gaussian random rough surface is proposed. Then, the sectional Romberg integration based on a novel matrix method is applied to numerically solve the proposed model. Influences of the index of refraction $n$ and the RMS roughness $\sigma_{r m s}$ on the emissivity $\varepsilon$ are analyzed and discussed. Experimental verification is carried out with Inconel 718 samples. In this research, diffraction is neglected, and the differences between real [36] and Gaussian rough surface are also neglected. Their potential effects on emissivity are to be further researched. The main conclusions drawn from this work are as follows:

(1) A directional spectral emissivity prediction model of rough surfaces is established considering the shadowing effect and the reflection enhancement. Sectional Romberg integration based on a novel matrix method is applied to numerically solve the proposed model and is theoretically verified. The computational time of the matrix method is compared with that of the traditional method. Errors induced in the matrix method are estimated.

(2) The influence of the index of refraction $n$ and RMS roughness $\sigma_{r m s}$ on the emissivity $\varepsilon$ is discussed according to the model. It is found that higher order components are smaller in scale and their energy is more concentrated near the normal direction. 
(3) $n$ influences $\varepsilon$ by influencing $\varepsilon_{F}$ (or from an optics point of view, the emission process). When $\sigma_{r m s}$ is relatively low, $\varepsilon$ tends to decrease with increasing $n$, and have a steeper peak in high viewing angles. The extreme point is the Brewster's angle, which increases with increasing $n$. When the RMS roughness $\sigma_{r m s}$ is relatively high, $\varepsilon$ would be dominated by $\sigma_{r m s}$, and influence of $n$ would be quenched.

(4) $\sigma_{r m s}$ influences both $S\left(\theta_{S}, \sigma_{r m s}\right)$ and emi $\left(\theta_{S}, n, \sigma_{r m s}\right)$ (both emitting and shadowing). That is, $\sigma_{r m s}$ effects both of emitting and shadowing. $\varepsilon$ decreases with increasing $\sigma_{r m s}$ in high viewing angle, increases with increasing $\sigma_{r m s}$ in middle viewing angle, and first increases then decreases with increasing $\sigma_{r m s}$ near normal direction. Surfaces with very high $\sigma_{r m s}$ may have a peak in low viewing angle.

(5) Surface with lower $\sigma_{r m s}$ and higher $n$, due to Fresnel's law, tends to have a peak in high viewing angles. On the contrary, surface with higher $\sigma_{r m s}$ and lower $n$, mainly due to reflection enhancement, tends to have a peak in low viewing angles. In the intermediate region, due to their combined effect, the emissivity has no recognizable peak and decreases with increasing viewing angle.

Author Contributions: Conceptualization, Q.S. and Z.L.; Methodology, J.H.; Software, J.H.; Validation, J.H. and J.Z.; Formal Analysis, J.H. and B.W.; Investigation, J.H.; Resources, Z.L., J.Z. and B.W.; Data Curation, J.H.; Writing-Original Draft Preparation, J.H.; Writing—Review \& Editing, Z.L., J.Z. and B.W.; Visualization, J.H.; Supervision, Z.L.; Project Administration, Z.L.; Funding Acquisition, Z.L. All authors have read and agreed to the published version of the manuscript.

Funding: This research was funded by the National Key Research and Development Program of China (2019YFB2005401), the National Natural Science Foundation of China (No. 91860207) and the Taishan Scholar Foundation and Shandong Provincial Key Research and Development Program (Major Scientific and Technological Innovation Project) (No.2020CXGC010204).

Institutional Review Board Statement: Not applicable.

Informed Consent Statement: Not applicable.

Data Availability Statement: The data presented in this study are available on request from the corresponding author.

Acknowledgments: The authors also gratefully acknowledge the support of the High Performance Machining Research Center at Shandong University, Jinan, China.

Conflicts of Interest: The authors declare no conflict of interest.

\section{Appendix A}

As a variable step-size integration method, Romberg integration is fast to converge and reliable in complex integration. It is defined as follows:

$$
\begin{gathered}
T_{n}=\frac{h}{2}\left[f(a)+f(b)+2 \sum_{k=1}^{n-1} f\left(x_{k}\right)\right] \\
S_{n}=T_{2 n}+\frac{1}{3}\left(T_{2 n}-T_{n}\right) \\
C_{n}=S_{2 n}+\frac{1}{15}\left(S_{2 n}-S_{n}\right) \\
R_{n}=C_{2 n}+\frac{1}{3}\left(C_{2 n}-C_{n}\right)
\end{gathered}
$$

where $h$ is the step-size, and $x_{K}$ are values of $x$ at the bisection points of the integration interval. Thus, $x_{1}, x_{17}$ are the upper and lower limit of integration, respectively. $R_{n}$ is the Romberg integration. $T_{n}, S_{n}, C_{n}$ are trapezoidal, Simpson and Cotes integration, respectively. Its error is estimated as:

$$
e_{R_{2 n}}=\left|R_{2 n}-R_{n}\right|
$$


In this study, the integration region is $(-\pi, \pi)$. In order to gain better accuracy, the region is divided into six equal segments. $n$ is set as 2 . In the first segment, which is $(-\pi,-2 \pi / 3)$, according to Equation (A1a) to Equation (A1d), we have:

$$
\begin{array}{r}
\int_{x_{1}}^{x_{17}} f(x) d x \quad \begin{array}{r}
\approx \\
R_{2}=
\end{array} \frac{x_{17}-x_{1}}{11,340}\left(217 x_{1}+1024 x_{2}+352 x_{3}+1024 x_{4}+436 x_{5}\right. \\
+1024 x_{6}+352 x_{7}+1024 x_{8}+434 x_{9}+1024 x_{10}+352 x_{11} \\
\left.+1024 x_{12}+436 x_{13}+1024 x_{14}+352 x_{15}+1024 x_{16}+217 x_{17}\right) \\
\int_{x_{1}}^{x_{17}} f(x) d x \approx R_{1}=\frac{x_{17}-x_{1}}{5670}\left(217 x_{1}+1024 x_{3}+352 x_{5}+1024 x_{7}\right. \\
\left.+436 x_{9}+1024 x_{11}+352 x_{13}+1024 x_{15}+217 x_{17}\right)
\end{array}
$$

Define:

$$
\begin{aligned}
& \operatorname{Rbg}_{2}^{\prime}=\frac{x_{17}-x_{1}}{11,340}\left[\begin{array}{llllllll}
217 & 1024 & 352 & 1024 & 436 & 1024 & 352 & 1024
\end{array}\right. \\
& \left.\begin{array}{lllllllll}
434 & 1024 & 352 & 1024 & 436 & 1024 & 352 & 1024 & 217
\end{array}\right] \\
& R b g_{1}^{\prime}=\frac{x_{17}-x_{1}}{5670}\left[\begin{array}{lllllllll}
217 & 0 & 1024 & 0 & 352 & 0 & 1024 & 0 \\
436 & 0 & 1024 & 0 & 352 & 0 & 1024 & 0 & 217
\end{array}\right] \\
& X^{\prime}=\left[\begin{array}{lllll}
x_{1} & x_{2} & x_{3} & \ldots & x_{17}
\end{array}\right]
\end{aligned}
$$

thus:

$$
\begin{aligned}
& R b g_{1}^{\prime} \cdot X^{\prime}=R_{1} \approx \int_{x_{1}}^{x_{17}} f(x) d x \\
& R b g_{2}^{\prime} \cdot X^{\prime}=R_{2} \approx \int_{x_{1}}^{x_{17}} f(x) d x
\end{aligned}
$$

In this study, sectional Romberg integration is imposed. We define:

$$
\begin{aligned}
& R b g_{2}=\frac{\pi}{3 \cdot 11,340}\left[\begin{array}{llllll}
217 & R b g^{\prime} & 434 & R b g^{\prime} & 434 & R b g^{\prime}
\end{array}\right.
\end{aligned}
$$

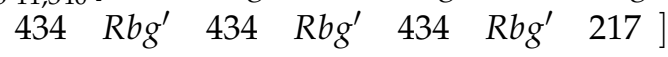

$$
\begin{aligned}
& \text { Rbg }^{\prime}=\left[\begin{array}{lllllll}
1024 & 352 & 1024 & 436 & 1024 & 352 & 1024
\end{array}\right. \\
& \left.\begin{array}{llllllll}
434 & 1024 & 352 & 1024 & 436 & 1024 & 352 & 1024
\end{array}\right] \\
& R b g_{1}=\frac{\pi}{3 \cdot 11,340}\left[\begin{array}{llllll}
217 & R b g^{\prime \prime} & 434 & R b g^{\prime \prime} & 434 & R b g^{\prime \prime}
\end{array}\right. \\
& 434 \quad R^{\prime \prime \prime} \quad 434 \quad R^{\prime \prime} g^{\prime \prime} \quad 434 \quad R^{\prime \prime \prime} g^{\prime \prime} \quad 217 \text { ] } \\
& \mathrm{Rbg}^{\prime}=\left[\begin{array}{lllllll}
0 & 1024 & 0 & 352 & 0 & 1024 & 0
\end{array}\right. \\
& 436 \quad 0 \quad 1024 \quad 0 \quad 352 \quad 0 \quad 1024 \quad 0] \\
& X=\left[\begin{array}{llllll}
f(-\pi) & f\left(\frac{-47 \pi}{48}\right) & f\left(\frac{-46 \pi}{48}\right) & \ldots & f\left(\frac{47 \pi}{48}\right) & f(\pi)
\end{array}\right]
\end{aligned}
$$

then:

$$
\begin{gathered}
\operatorname{Rbg}_{2} \cdot X \approx \int_{-\pi}^{\pi} f(x) d x \\
R b g_{1} \cdot X \approx \int_{-\pi}^{\pi} f(x) d x \\
e_{R_{2}}=\left|R b g_{2} \cdot X-R b g_{1} \cdot X\right|
\end{gathered}
$$

noting that $S\left(\theta_{S}, \sigma_{r m s}\right)$ is not a function of $p, q, \theta_{1}, \theta_{2}$ or $\phi$. Thus:

$$
\begin{aligned}
\operatorname{Der}_{k}(0)= & E m i \cdot S=\int_{-\infty}^{\cot \theta_{s}} \int_{-\infty}^{\infty} t\left(\theta_{s_{k}} p, q, \sigma_{r m s}\right) \varepsilon_{l}(n, p, q) s\left(\theta_{s_{k}}, \sigma_{r m s}\right) d p d q \approx \varepsilon_{0} \\
\operatorname{Der}_{k}(1) & =E m i \cdot(1-S) \cdot R b g_{2} \cdot r f t \cdot S \\
& =\int_{0}^{2 \pi} \int_{0}^{\pi-\theta_{s}} t\left(\theta_{s}, \theta_{1}, \phi_{1}, \sigma_{r m s}\right) S\left(\theta_{s}, \sigma_{r m s}\right)\left[1-\varepsilon_{l}\left(n, \theta_{1}, \phi_{1}\right)\right] \cdot \sin \theta_{1} \\
& \cdot \int_{-\infty}^{\cot \theta_{1}} \int_{-\infty}^{\infty} t\left(\theta_{1}, p_{1}, q_{1}, \sigma_{r m s}\right)\left[1-S\left(\theta_{1}, \sigma_{r m s}\right)\right] \varepsilon_{l}\left(n, p_{1}, q_{1}\right) d p_{1} d q_{1} d \theta_{1} d \phi_{1} \approx \varepsilon_{1}
\end{aligned}
$$


High-order components could be verified similarly. The error was estimated according to Equation (A10).

\section{References}

1. Alaruri, S.; Bianchini, L.; Brewington, A. Effective spectral emissivity measurements of superalloys and YSZ thermal barrier coating at high temperatures using a $1.6 \mu \mathrm{m}$ single wavelength pyrometer. Opt. Lasers Eng. 1998, 30, 77-91. [CrossRef]

2. Kong, B.; Li, T.; Eri, Q. Normal spectral emissivity measurement on five aeronautical alloys. J. Alloys Compd. 2017, 703, 125-138. [CrossRef]

3. González-Fernández, L.; Risueño, E.; Pérez-Sáez, R.B.; Tello, M.J. Infrared normal spectral emissivity of Ti-6Al-4V alloy in the 500-1150 K temperature range. J. Alloys Compd. 2012, 541, 144-149. [CrossRef]

4. Tanda, G.; Misale, M. Measurement of total hemispherical emittance and specific heat of aluminum and inconel 718 by a calorimetric technique. J. Heat Transf. 2006, 128, 302-306. [CrossRef]

5. Keller, B.P.; Nelson, S.E.; Walton, K.L.; Ghosh, T.K.; Tompson, R.V.; Loyalka, S.K. Total hemispherical emissivity of inconel 718. Nucl. Eng. Des. 2015, 287, 11-18. [CrossRef]

6. Cagran, C.P.; Hanssen, L.M.; Noorma, M.; Gura, A.V.; Mekhontsev, S.N. Temperature-resolved infrared spectral emissivity of SiC and Pt-10Rh for temperatures up to $900{ }^{\circ} \mathrm{C}$. Int. J. Thermophys. 2007, 28, 581-597. [CrossRef]

7. Abbott, W.; Cook, C.R.; Garfield, G.; Barice, W.J. Titanium and Titanium Alloys; Matthew, J.D., Ed.; American Society for Metals: Geauga County, $\mathrm{OH}, \mathrm{USA}, 1982$.

8. Xu, D.; Wang, Y.; Xiong, B.; Li, T. MEMS-based thermoelectric infrared sensors: A review. Front. Mech. Eng. 2017, 12, 557-566. [CrossRef]

9. Ezugwu, E.O.; Wang, Z.M.; Machado, A.R. The machinability of nickel-based alloys: A review. J. Mater. Process. Technol. 1998, 86, 1-16. [CrossRef]

10. González de Arrieta, I.; González-Fernández, L.; Risueño, E.; Echániz, T.; Tello, M.J. Isothermal oxidation kinetics of nitrided Ti-6Al-4V studied by infrared emissivity. Corros. Sci. 2020, 173, 108723. [CrossRef]

11. Qi, J.; Eri, Q.; Kong, B.; Zhang, Y. The radiative characteristics of inconel 718 superalloy after thermal oxidation. J. Alloys Compd. 2020, 156414. [CrossRef]

12. Frolec, J.; Králík, T.; Musilová, V.; Hanzelka, P.; Srnka, A.; Jelínek, J. A database of metallic materials emissivities and absorptivities for cryogenics. Cryogenics 2019, 97, 85-99. [CrossRef]

13. Sabuga, W.; Todtenhaupt, R. Effect of Roughness on the Emissivity of the Precious Metals Silver, Gold, Palladium, Platinum, Rhodium, and Iridium. High Temp. High Press. 2001, 33, 261-269. [CrossRef]

14. Rubanenko, L.; Schorghofer, N.; Greenhagen, B.T.; Paige, D.A. Equilibrium temperatures and directional emissivity of sunlit airless surfaces with applications to the moon. J. Geophys. Res. Planets 2020, 125. [CrossRef]

15. Wen, C.-D.; Mudawar, I. Modeling the effects of surface roughness on the emissivity of aluminum alloys. Int. J. Heat Mass Transf. 2006, 49, 4279-4289. [CrossRef]

16. Chen, K.S.; Wu, T.D.; Tsang, L.; Li, Q.; Shi, J.; Fung, A.K. Emission of rough surfaces calculated by the integral equation method with comparison to three-dimensional moment method simulations. IEEE Trans. Geosci. Remote Sens. 2003, 41, 90-101. [CrossRef]

17. Wu, X.; Smith, W.L. Emissivity of rough sea surface for $8-13 \mu \mathrm{m}$ : Modeling and verification. Appl. Opt. 1997, 36, 2609-2619. [CrossRef] [PubMed]

18. Li, H.; Pinel, N.; Bourlier, C. Polarized infrared reflectivity of 2D sea surfaces with two surface reflections. Remote Sens. Environ. 2014, 147, 145-155. [CrossRef]

19. Li, H.; Pinel, N.; Bourlier, C. Polarized infrared reflectivity of one-dimensional gaussian sea surfaces with surface reflections. Appl. Opt. 2013, 52, 6100-6111. [CrossRef]

20. Li, H.; Pinel, N.; Bourlier, C. A monostatic illumination function with surface reflections from one-dimensional rough surfaces. Waves Random Complex Media 2011, 21, 105-134. [CrossRef]

21. Li, H.; Pinel, N.; Bourlier, C. Polarized infrared emissivity of 2D sea surfaces with one surface reflection. Remote Sens. Environ. 2012, 124, 299-309. [CrossRef]

22. Tang, K.; Buckius, R.O. A statistical model of wave scattering from random rough surfaces. Int. J. Heat Mass Transf. 2001, 44, 4059-4073. [CrossRef]

23. $\mathrm{Hu}, \mathrm{Y} . Z$.; Tonder, K. Simulation of 3D random rough surface by 2D digital filter and fourier analysis. Int. J. Mach. Tools Manuf. 1992, 32, 83-90. [CrossRef]

24. Smith, B.G. Geometrical shadowing of a random rough surface. IEEE Trans. Antennas Propag. 1967, 15, 668-671. [CrossRef]

25. Smith, B.G. Lunar surface roughness: Shadowing and thermal emission. J. Geophys. Res. 1967, 72, 4059-4067. [CrossRef]

26. Bourlier, C.; Berginc, G.; Saillard, J. Monostatic and bistatic statistical shadowing functions from a one-dimensional stationary randomly rough surface according to the observation length: I. Single scattering. Waves Random Media 2002, 12, 145-173. [CrossRef]

27. Yoshimori, K.; Itoh, K.; Ichioka, Y. Optical characteristics of a wind-roughened water surface: A two-dimensional theory. Appl. Opt. 1994, 34, 6236-6247. [CrossRef] [PubMed]

28. Born, M. Principles of Optics: Electromagnetic Theory of Propagation, Interference, and Diffraction of Light, 6th ed.; Wolf, E., Ed.; Pergamon Press: Oxford, UK, 1980. 
29. Balat-Pichelin, M.; Bousquet, A. Total hemispherical emissivity of sintered SiC up to $1850 \mathrm{~K}$ in high vacuum and in air at different pressures. J. Eur. Ceram. Soc. 2018, 38, 3447-3456. [CrossRef]

30. Brodu, E.; Balat-Pichelin, M.; Sans, J.L.; Kasper, J.C. Influence of roughness and composition on the total emissivity of tungsten, rhenium and tungsten-25\% rhenium alloy at high temperature. J. Alloys Compd. 2014, 585, 510-517. [CrossRef]

31. Tang, K.; Buckius, R.O. The geometric optics approximation for reflection from two-dimensional random rough surfaces. Int. J. Heat Mass Transf. 1998, 41, 2037-2047. [CrossRef]

32. Brodu, E.; Balat-Pichelin, M.; Sans, J.L.; Freeman, M.D.; Kasper, J.C. Efficiency and behavior of textured high emissivity metallic coatings at high temperature. Mater. Des. 2015, 83, 85-94. [CrossRef]

33. Zhao, J.; Liu, Z. Influences of coating thickness on cutting temperature for dry hard turning inconel 718 with PVD TiAlN coated carbide tools in initial tool wear stage. J. Manuf. Process. 2020, 56, 1155-1165. [CrossRef]

34. Zhao, J.; Liu, Z.; Wang, B.; Hua, Y.; Wang, Q. Cutting temperature measurement using an improved two-color infrared thermometer in turning inconel 718 with whisker-reinforced ceramic tools. Ceram. Int. 2018, 44, 19002-19007. [CrossRef]

35. Del Campo, L.; Pérez-Sáez, R.B.; González-Fernández, L.; Esquisabel, X.; Fernández, I.; González-Martín, P.; Tello, M.J. Emissivity measurements on aeronautical alloys. J. Alloys Compd. 2010, 489, 482-487. [CrossRef]

36. Țălu, Ș. Micro and Nanoscale Characterization of Three Dimensional Surfaces: Basics and Applications; Napoca Star Publishing House: Cluj-Napoca, Romania, 2015. 